\title{
Differences in responses to breakfast between breakfast eaters and breakfast skippers
}

\author{
S. Reeves ${ }^{1}$, J. Huber ${ }^{2}$, T. Smith ${ }^{1}$, M. Villegas-Montes ${ }^{1}$, J. Elgumati ${ }^{1}$ and L. Halsey ${ }^{1}$ \\ ${ }^{1}$ Health Sciences Research Centre, University of Roehampton, London, SW15 4JD and ${ }^{2}$ University of Northampton, \\ Park Campus, Boughton Green Road, Northampton, UK, NN2 7AL, UK
}

Studies have shown that body mass index (BMI) in breakfast eaters is typically lower than that of breakfast skippers either as a result of differences in energy intake ${ }^{(1)}$ or energy expenditure $(\mathrm{EE})^{(2)}$. Since evidence available is unable to clarify the mechanisms that link BMI with regular breakfast consumption ${ }^{(3)}$, our study aimed to explain this association with an experimental trial to establish underlying differences between breakfast eaters and breakfast skippers that were normal weight and overweight, by assessing resting metabolic rate (RMR), post-breakfast EE, blood glucose, hunger, physical activity and 'morningness' (an indicator of personal circadian rhythm). Ethical approval was granted by the University of Roehampton.

Data were collected from 37 participants and were organised into four groups: Group 1 low BMI breakfast eater, Group 2 low BMI breakfast skipper, Group 3 high BMI breakfast eater, Group 4 high BMI breakfast skipper. Douglas bags, blood samples and hunger ratings were collected at baseline and every 30 min for 3 hours following a carbohydrate based breakfast. Physical activity and food intake were assessed with seven day diaries and morningess using the Composite Morningess questionnaire ${ }^{(4)}$.

\begin{tabular}{|c|c|c|c|c|c|c|c|c|c|c|c|c|}
\hline \multirow[b]{2}{*}{ Group } & \multicolumn{2}{|c|}{ BMI $\left(\mathrm{kg} / \mathrm{m}^{2}\right)$} & \multicolumn{2}{|c|}{ RMR (kcal/kg/day) } & \multicolumn{2}{|c|}{ EE post-prandial (kcal/kg/day) } & \multicolumn{2}{|c|}{ Glucose $\mathrm{AUC}_{165 \mathrm{~min}}(\mathrm{mmol} . \mathrm{min} / \mathrm{l})$} & \multicolumn{2}{|c|}{ Hunger $(\%)$} & \multicolumn{2}{|c|}{ Physical activity (MET $\dagger$-min/week) } \\
\hline & Mean & SD & Mean & SD & Mean & SD & Mean & SD & Mean & SD & Mean & SD \\
\hline 1 & 21.6 & 1.3 & 24.5 & 3.4 & 30.0 & 3.8 & 922.1 & 91.0 & 65.9 & 16.6 & 3475 & 2723 \\
\hline 2 & 21.1 & 2.2 & 23.8 & 4.6 & 31.6 & 4.0 & 931.1 & 115.8 & $16.0^{\mathrm{a}}$ & 17.4 & 3181 & 1374 \\
\hline 3 & $30.5^{* *}$ & 7.7 & $19.6^{*}$ & 2.8 & $23.5^{\mathrm{b}}$ & 3.7 & 974.7 & 103.6 & 37.9 & 26.4 & 2944 & 1663 \\
\hline 4 & $28.7^{* *}$ & 3.3 & 20.8 & 3.2 & $24.4^{\mathrm{b}}$ & 3.9 & 985.1 & 132.5 & $33.2^{\mathrm{a}}$ & 30.1 & 4815 & 3616 \\
\hline
\end{tabular}

${ }^{\dagger}$ Metabolic equivalent task. ${ }^{*}$ Groups $3 \& 4$ greater than $1 \& 2(p<0.01), * 3$ lower than $1(p<0.05),{ }^{\mathrm{a}} 2 \& 4$ lower than $1(p<0.01),{ }^{\mathrm{b}} 3 \& 4$ lower than $1 \& 2(p<0.01)$.

The overweight groups $3 \& 4$ had higher BMI's, lower RMR and lower EE (both) per kg per day than the normal BMI groups $1 \& 2$. There were no significant differences in energy intakes between groups. Breakfast skippers were notably less hungry during the morning $(P=0.01)$, consumed more caffeine per day $(P=0.04)$ and also tended to show less morningness $(P=0.06)$; thus it is possible that there are physiological and behavioural predispositions that determine breakfasting habits. Further work will compare all groups in both breakfast and non-breakfast conditions.

This work was supported by Kellogg's.

1. Utter J, Scragg R, Mhurchu C et al. (2007) J Am Diet Assoc 107, 570-576.

2. Timlin M, Pereira MA, Story M et al. (2008) Pediatrics 121, e638-645.

3. Halsey L, Huber J, Reeves S et al. (2012) Pub J Health Nutr 15, 238-245.

4. Barton J, Costa G, Smith L et al. (1995) Work \& Stress 9, 4-30. 